\title{
LA RED SOCIOTÉCNICA ORIGINADA EN COSTA RICA, QUE PERMITIÓ EL DESARROLLO DEL PRIMER SUERO ANTIOFIDICO POLIVALENTE PARA ÁFRICA SUBSAHARIANA
}

\section{THE SOCIO-TECHNICAL NETWORK ORIGINATED IN COSTA RICA, WHICH ALLOWED THE DEVELOPMENT OF THE FIRST POLYVALENT ANTIVENOM FOR SUB-SAHARAN AFRICA}

\author{
Ana Lucía Calderón Saravia*
}

RESUMEN

Este artículo trata un estudio de caso de una comunidad tecnocientífica, el Instituto Clodomiro Picado Twight y el desarrollo del primer suero antiofídico polivalente para África Subsahariana. Pretende aportar algunos elementos teórico conceptuales generados desde las investigaciones sociales de la ciencia y la tecnología o la denominada corriente Ciencia, Tecnología y Sociedad (стs), para el estudio de comunidades científicas y tecnológicas, su producción de conocimiento y artefactos, así como algunos aportes desde la Antropología.

PALABRAS CLAVE: COSTA RICA * CIENCIA Y TECNOLOGÍA * SUERO ANTIOFÍDICO * ANTROPOLOGÍA $*$ SOCIEDAD

\section{ABSTRACT}

This article discusses a case study of a scientific techno community, the Instituto Clodomiro Picado Twight and development of the first polyvalent antivenom for subSaharan Africa. It aims to provide some conceptual and theoretical elements generated from social research of science and technology or current called Science, Technology and Society (STS) for the study of scientific and technological communities, their production of knowledge and artifacts, as well as some contributions from Anthropology.

KEYWORDS: COSTA RICA * SCIENCE AND TECHNOLOGY * POLYVALENT ANTIVENOM * ANTHROPOLOGY * SOCIETY

* Departamento de Investigación y Evaluación Académica (DIEA-UCR), Escuela de Antropología de la Universidad de Costa Rica (UCR).

ana.calderon@ucr.ac.cr 


\section{PRESENTACIÓN}

Este trabajo es producto de una investigación realizada en el Instituto Clodomiro Picado Twight (IсP), de la Facultad de Microbiología de la Universidad de Costa Rica, el cual se dedica a la investigación principalmente de serpientes, sus venenos, sus efectos y mecanismos para neutralizarlos por medio de la elaboración de sueros antiofídicos. Tanto personal ${ }^{1}$ de la División Académica, dedicada a investigación básica, como de la División Industrial, dedicada a investigación aplicada para la elaboración de sueros, aportaron con sus testimonios la información que permitió reconstruir la red que se analiza en este artículo. La información que permite reconstruir el proceso de conformación de la red sociotécnica para el desarrollo de una nueva variedad de suero, se presenta apoyándose en los planteamientos de la Teoría del ActorRed (TAR) y su método.

\section{FUNDAMENTOS TEÓRICOS Y METODOLOGÍA UTILIZADA}

La TAR denominada también como Sociología de la Traducción, aporta los elementos teóricos de partida para este trabajo. Se considera de gran utilidad porque incluye en el análisis de las redes tanto humanos como no humanos, analizando de manera simétrica su papel en las relaciones que son las que generan la red pero al mismo tiempo se crean y recrean en ella, integrando objetos tanto naturales como artificiales o construidos socialmente, $y$ se deja de concebir a los no humanos como entes pasivos $y$ se les asigna un rol activo dentro de las redes. Al respecto, Ortega y Arellano (2010) señalan que en el estudio de comunidades científicas, las fronteras entre sociedad y naturaleza se disuelven, pues características que antes podían ser atribuidas únicamente

$1 \quad$ Este trabajo fue realizado gracias al apoyo del personal del Instituto Clodomiro Picado Twight. Es importante señalar que se le ofreció confidencialidad a las personas que aportaron información para este trabajo, de modo que en algunos casos se hará referencia a quién aportó los datos y en otros casos se señalará la fecha y el número con el que fue clasificada cada una de las personas que colaboraron. a seres humanos, en el ámbito científico aparecen relacionadas con elementos que no son humanos, de modo que explicar la realidad a partir de la dicotomía entre las nociones de lo social y lo natural dejan de ser útiles.

Las proposiciones de Ortega y Arellano parten de esta Sociología de la Traducción, propuesta por Latour (2007), que rompe con las dicotomías naturaleza-sociedad, sujeto-objeto, macro-micro y humano-no humano, para realizar investigaciones sobre la naturaleza, la ciencia y la tecnología desde una perspectiva social. La traducción supone entonces el análisis de elementos y recursos que, aunque disímiles $y$ heterogéneos, mediante un ejercicio de equivalencia permite estudiar sus relaciones $y$ cómo funcionan juntos. Dentro de la propuesta de Latour (1996), quienes participan en la red no se les denomina actores, sino más bien "actantes" , un término más neutro.

Asimismo, Martin (2000) (citado en Ortega, 2013) y Arellano (1999) presentan cuatro momentos para la metodología de la TAR:

1) La problematización: los científicos procuran definir el objeto o la cuestión de la investigación, de manera que otros actores se interesen en ese objeto o en esa cuestión y acoten su definición.

2) La participación: los investigadores sellan alianzas con los actores asociados a la problemática, construyen su sistema de alianzas formando estructuras sociales específicas e interrumpiendo eventuales asociaciones competidoras.

3) El enrolamiento: es el mecanismo por el cual un rol es definido $y$ atribuido a un actor que lo acepta. Ese rol no se halla necesariamente predefinido, sino que es progresivamente construido y estabilizado durante esta etapa.

$2 \quad$ El/la "actante" es una definición semiótica que se refiere no solo a "alguien" sino a "algo" que actúa o cuya actividad es asumida por otros(as), lo que implica no solo la actuación de humanos sino que incluso estos pueden estar ausentes de la acción. Así, un actante puede ser literalmente cualquier cosa provista de lo que sería esta fuente de acción garantizada. 
4) La movilización: los diferentes actores se asocian unos con otros a través de sus voceros. La designación de voceros permite reducir el número de interlocutores $y$ homogeneizar los puntos de vista, los intereses $y$ los lenguajes; permite entonces convertir grupos heterogéneos $y$ numerosos en una cantidad de entidades homogéneas y por lo tanto, traducciones encaminadas a representar socialmente al conjunto de actores (Martin, 2001 y Arellano, 1999).

La definición de estos cuatro pasos permite a su vez identificar cómo se construye una red, el problema que sirve de punto de partida para el involucramiento de los distintos actantes, qué actantes participan en ella y cómo se conectan unos y otros, las negociaciones que llevan a cabo, quién tiene el poder y cómo lo ejerce, las intervenciones que los distintos participantes en la red hacen sobre los objetos técnicos y naturales y la sociedad, así como, los acuerdos a los que llegan sustentados en la base material de actantes no humanos involucrados.

La búsqueda de respuestas o de soluciones para un problema motiva el inicio de la red $y$ a partir de los intereses de distintos actantes, deciden involucrarse y cumplir con un rol que no es estable sino que va configurándose a partir de la conexión con otros (as) actantes $y$ las expectativas de unos $y$ otros sobre el rol que cada uno (a) debe cumplir, para así lograr la interacción simétrica entre entidades diversas y heterogéneas. Dentro de las redes, los seres humanos tienen una doble función: por un lado, hacen los trazos de la red al mismo tiempo que se inscriben en ella. Por lo tanto, las redes son entidades que evidencian trazos, trayectorias, pero también conexiones e inscripciones de quienes participan en las relaciones que se establecen, en este caso, para la producción de conocimiento científico o de tecnología (Latour, 1996).

Por medio de las técnicas de entrevista, observación y análisis de discurso, se recuperó la información que permitió reconstruir el proceso de conformación de la red sociotécnica, que tuvo como resultado el desarrollo de una nueva variedad de suero antiofídico, producido en Costa Rica para tratar a víctimas de mordeduras de serpientes en países de África Subsahariana.

\section{ALGUNOS DATOS DE LA COMUNIDAD DE ESTUDIO}

El Instituto Clodomiro Picado Twight ${ }^{3}$ (IcP) se creó el 13 de abril de 1970 como una dependencia del Ministerio de Salubridad Pública, en convenio con la Facultad de Microbiología de la Universidad de Costa Rica, siendo el personal técnico y académico de esta universidad quien apoya su instalación. Dos años después, el 2 de junio de 1972, el Ministerio traslada el IcP completamente a la Universidad, mediante la firma de un convenio (Gutiérrez, Segura y Aymerich, 1991). Si bien, el objetivo primordial de su creación fue que el país y la región contaran con un laboratorio para la producción de sueros antiofídicos, desde sus inicios su primer director, el Dr. Róger Bolaños impulsó la conformación de un equipo académico que realizara estudios de posgrado en el exterior, que se dedicara a la investigación científica y que publicara sus resultados en revistas científicas internacionales. Así se creó una cultura que prevalece hasta la actualidad, que combina producción de conocimiento por medio de investigaciones científicas y un programa de acción social ${ }^{4}$ para capacitar al personal de salud de zonas de Costa Rica con alta incidencia de accidentes ofídicos, principalmente comunidades

$3 \quad$ El Dr. Clodomiro Picado Twight (1887-1944) fue un médico y científico costarricense que investigó y se interesó por una gran cantidad de temas, entre ellos los accidentes ofídicos, el efecto del veneno de serpientes en las personas y los mecanismos para neutralizarlos utilizando sueros antiofídicos. Es el autor del libro "Serpientes venenosas de Costa Rica: Seroterapia antiofídica”, el cual se publicó en 1931 e impulsó la creación de la "Ley de Defensa contra el Ofidismo" en 1929, aspecto que contribuyó a que las mordeduras por serpientes venenosas fueran consideradas en Costa Rica un problema de salud pública (Gutiérrez, 2010).

4 La acción social es la vía mediante la cual la Universidad de Costa Rica se vincula con la sociedad del país por medio de actividades y proyectos de extensión cultural, así como docente e investigación aplicada. 
rurales pobres, además de la producción de sueros - que como se describe más adelante-al final del siglo xx enfrentaba serias dificultades.

\section{EL PRIMER RETO: LA CENTROAMERICANIZACIÓN DEL ICP Y LA INNOVACIÓN TECNOLÓGICA}

A mediados de la década de 1990, luego de más de 20 años de ser el único laboratorio de producción de sueros antiofídicos ${ }^{5}$ en el país y en la región centroamericana, el IcP tenía serios problemas para producir el objeto que motivó su creación. Uno tras otro, los lotes de suero debían desecharse porque estaban contaminados y producían fiebre, imposible utilizarlos en seres humanos y en animales ¿Qué estaba pasando? ¿Por qué tantos años de experiencia no eran suficientes para lograr la calidad que se quería en los sueros? En efecto, el ICP venía produciendo suero pero había dos aspectos importantes que impedían dar un salto cuantitativo y cualitativo en la producción. A principios de los años 90 del siglo Xx, el ICP se organizaba en una División de Producción y una División de Investigación, asimismo, las decisiones se tomaban en tres consejos: el Directivo, que hoy vendría a ser el Consejo Asesor que apoya a la dirección; un Consejo de Investigación, que en la actualidad es el Consejo Científico, que toma decisiones sobre el trabajo académico; $y$ un Consejo de Producción, que en este momento sería el Consejo Industrial, que organiza la producción de sueros.

El señor Abel Robles estaba al frente de la entonces llamada División de Producción, tenía una gran experiencia en la producción de sueros y trabajó con el primer director del ICP, el Dr. Róger Bolaños. Pese a sus muchos años de trabajo, no lograba identificar los aspectos que intervenían en la pérdida de una gran cantidad de lotes de suero debido a su mala calidad.

$5 \quad$ Según Gutiérrez, el suero se produce mediante la inmunización activa de caballos adultos sanos con dosis determinadas de venenos de serpiente. El caballo desarrolla anticuerpos, se le extrae sangre, la cual es purificada mediante procesos de laboratorio para separar del plasma los anticuerpos $y$ proteínas que constituyen la materia prima para la elaboración del suero (c.p. dic., 2011).
Robles era un técnico que no contaba con estudios formales en Microbiología o Química, pero conocía muy bien los procesos necesarios para producir los sueros y al igual que la mayoría del personal, se sentía identificado con esta producción que ayuda a salvar a víctimas de accidentes ofídicos de zonas rurales pobres.

Por otro lado, en la División de Investigación - ahora División Académica-, se ubicaba la Sección de Desarrollo Tecnológico, la cual debía apoyar el mejoramiento de la producción de suero; no obstante, el personal de investigación no logró incidir realmente en este ámbito. La elaboración de sueros antiofídicos no atraía al personal de investigación, cuya prioridad era la investigación básica. Aunque conscientes del impacto social del suero antiofídico y de su importancia para salvar la vida de muchas personas, puede deducirse, a partir de las entrevistas realizadas, que las y los investigadores no visualizaban la actividad productiva como una actividad sustantiva de su quehacer. El ICP se orientó principalmente a la investigación básica sobre temas relacionados con los efectos de venenos y sus toxinas o con biología de las serpientes de Costa Rica, lo cual se demuestra en las publicaciones científicas, esto pese a la conciencia social sobre lo que la producción de sueros representa.

Otro aspecto derivado de la no intervención del personal de investigación en la elaboración de sueros consistió en que la producción estaba en manos de personal técnico, quien tenía buena voluntad y compromiso, sin embargo, no tenían estudios formales que les permitieran identificar los mecanismos para producir los sueros con los mejores resultados, sino que trabajaban a partir de algunos conocimientos básicos, su intuición y sus creencias. En este escenario, los pedidos de suero se entregaban con retraso y el personal estaba frustrado, desanimado y sin la capacidad de entender lo que ocurría:

...empezaba un lote [de suero] y no tenía garantía de que ese lote llegara a buen término, usted iba jugando y conforme el proceso se iba quemando, se iba dando cuenta de si el lote pintaba 
bien o pintaba mal. Al final hacer una prueba, la pesadilla de todos nosotros eran los pirógenos, que es un derivado de un tipo de bacterias que contamina los productos inyectables, en el caso nuestro, el suero, $y$ en el paciente producen fiebre; piro de fuego, que producen fuego, fiebre. Cuando uno inyecta un suero pirogénico en una persona, ella desarrollará un cuadro febril y eso es inaceptable en un inyectable, entonces si el suero se contaminaba con bacterias $y$ quedaba pirogénico, (...) iba para la basura (...), no teníamos claras las razones por las cuales los sueros se contaminaban, no lo teníamos en nuestro control el proceso, o se nos escapaba qué era lo que teníamos mal (Guillermo León Montero, entrevista, 20/11/2013).

El Dr. Guillermo León se encontró esta situación al ingresar a trabajar en el IcP en 1994. Si bien es cierto, su trabajo inició como investigador, se acercó al señor Abel Robles para adquirir conocimientos sobre los sueros y su producción:

Yo no tenía respuesta, le preguntaba a Robles porque él sabía mucho más que yo de esto, él decía que tampoco tenía idea, que esta situación no pasaba por primera vez - ya ha pasado, no sabemos la causa y en algún momento deja de pasar, tenga paciencia, sepa que esto no es lo más importante que está pasando en su vida, aprenda a vivir con esto, porque esto es así, y tenga fe en que un día un lote va a salir bien-. Esas palabras me consolaron mucho porque él era el modelo que yo tenía y si él lo decía, seguramente era así (Guillermo León Montero, entrevista, 20/11/2013).

Si bien, el suero se producía en forma paralela a la investigación científica básica, el personal de investigación no abordaba este proceso, solamente participaba en ocasiones en la parte final, por ejemplo, etiquetando los frascos o viales de suero $y$ chequeando su pureza, viendo contra un fondo iluminado cada vial para cerciorarse que no tenía contaminantes visibles, empacando el pedido para enviarlo, pero no intervenían en las etapas previas. El proceso de producción de suero era diferente, incluso más artesanal, se utilizaban sales de sulfato de amonio para purificar el plasma y obtener así los anticuerpos contenidos en la sangre de los caballos; pero esta técnica tenía un costo económico elevado y además se tardaba entre 3-4 semanas para producir un lote de suero, que apenas tenía una capacidad neutralizante del 40\%, según recuerda el Dr. Gustavo Rojas, director del Icp entre los años 1996-2004.

Antes de ser director $y$ preocupado por la situación tan incierta de la producción de suero, el Dr. Rojas experimentó con una técnica que había sido probaba a nivel de laboratorio, pero él quería desarrollarla a nivel industrial: utilizar ácido caprílico para purificar el plasma. Esta innovación permitiría abaratar los costos de producción, además el suero tendría más pureza, lo que significa que contenía menos proteínas de caballo, característica que lo hacía un producto con una menor generación de reacciones alérgicas y con un rendimiento del $70 \%$ de capacidad neutralizante de los venenos. No obstante, lograr llevar la innovación del laboratorio a un nivel industrial tomó tiempo y muchos intentos fallidos hasta lograr el éxito en 1994, suceso que quedó plasmado en la publicación de Rojas, G., Jiménez, J.M. y Gutiérrez, J.M. titulado "Caprylic acid fractionation of hyperimmune horse plasma: description of a simple procedure for antivenom production".

Posterior a comprobar la factibilidad de la innovación, siguió una etapa para convencer al personal encargado de la producción de sueros en el uso del nuevo proceso; asimismo, se invirtió en infraestructura y equipos. El primer paso para los cambios siguientes ya se había dado, como apunta el Dr. Rojas, “...se alinearon los planetas...se dieron muchas circunstancias, muchas situaciones..." (Gustavo Rojas, entrevista, 26/03/2014).

En esa época, el director era el Dr. José María Gutiérrez, quién decidió separar la planta de producción de sueros del resto de la infraestructura del Instituto, aspecto que posteriormente tendría un impacto importante en 
los cambios de esta unidad. En su último año de gestión, se aprobó un reglamento oficial con la finalidad de guiar la organización y las acciones del IcP; asimismo, se decidió trasladar la Sección de Desarrollo Tecnológico, la cual se ubicada en la División Académica hacia la División Industrial.

En 1996, el Dr. Rojas asumió la dirección del IcP y fue convocado a una reunión de la Red del Sector Salud de Centroamérica y República Dominicana (Resscad), realizada en Panamá. En esa actividad, los entonces Ministros y Viceministros de Salud de los países miembros, designaron al ICP de la ucR, como laboratorio de referencia para la producción de sueros antiofídicos en la región, a partir de la discusión que se dio sobre el ofidismo y los 5000 accidentes ofídicos que ocurrían anualmente en esa época, sin que hubiera producción de suero, salvo en Costa Rica. Se había demostrado que los sueros del IcP podían neutralizar los envenenamientos producidos por las serpientes centroamericanas; pese al acuerdo que se firmó y a la aceptación del reto por parte del Dr. Rojas, la preocupación era poder satisfacer la demanda porque tenían serios problemas en la capacidad de producción. Si bien, el acuerdo firmado en Panamá por las autoridades gubernamentales en salud de la región, fue un motor para impulsar cambios y buscar mecanismos de mejora, también generó una controversia al interno del ICP y en el ámbito universitario:

...un acuerdo en el contexto de la salud, pero un acuerdo político al fin y al cabo (...), sirvió para pedir apoyo en la universidad (...) en 1994 el Clodomiro ${ }^{6}$ producía de 20 a 30 mil frascos al año, solo para el mercado nacional (...) el ácido caprílico ayudaba, pero no teníamos suficientes caballos (colaborador I-11, entrevista, 25/03/2014).

Los distintos grupos sociales $y$ actores presionan ahora el avance en la producción de suero. Países como Colombia y Ecuador, incluso Estados Unidos, contactaron también

$6 \quad$ El ICP es conocido popularmente por este nombre. al ICP para explorar la posibilidad de la venta de sueros. No obstante, los problemas en la producción no eran la única preocupación en ese momento. Al interior del icp se generó una ardua discusión porque la producción de suero no calzaba con los objetivos académicos de la unidad y surgió una controversia que parece persistir hasta la actualidad: producción de sueros versus investigación básica y académica.

Dentro de las posibilidades propuestas en la Universidad, se encontraba la de ceder la producción de sueros a una empresa privada; sin embargo, esa alternativa era contraria al espíritu de la acción social de la institución, porque los sueros debían prioritariamente salvar vidas de personas de escasos recursos de zonas rurales, eso era un deber y un compromiso, no podía verse como un negocio. De esto surgió la duda sobre si ampliar la producción de sueros $y$ darle una orientación empresarial era lo correcto. Finalmente, privó el carácter social de la Universidad $y$ del Instituto, debido a que la mayoría de personas afectadas provienen de zonas rurales pobres $y$ la falta de este medicamento podría provocar lesiones o la muerte. Por esta razón, había que ampliar la producción; no obstante, el problema se encontraba en cómo lograrlo, si de diez lotes de suero, solo dos funcionaban ¿qué podrían hacer para cumplir con el compromiso adquirido?

... si pretendíamos abarcar el mercado centroamericano teníamos que tener más dominio de lo que estábamos haciendo, entonces empezamos a hacer una revisión, paso por paso, de todos los procesos de producción y empezamos a encontrarle los "demonios que estaban escondidos" en el proceso, muchos de ellos asociados al mismo proceso de expansión de actividades, porque si en un momento hacíamos 30000 viales al año, ya ahora que queríamos venderle a Centroamérica entonces estábamos aspirando a hacer 40 o 50 mil (colaborador I-10, entrevista, 21/06/2013).

Las preocupaciones $y$ las dudas eran muchas, había que realizar inversiones, el personal tenía que enfrentar las nuevas demandas, realizar 
más trabajo, cambiar prácticas y creencias, asimismo tenían que lidiar con las serias deficiencias de la producción, había que adquirir más caballos, hacer más sangrías, es decir, más procesos de extracción de sangre.

... una operación que parecía fácil, realmente no lo era; punzar un caballo $y$ sacarle sangre ya es difícil, pero además sacarle sangre estéril es más difícil, y si usted considera que la sangre es una matriz en la que se cultivan bacterias... (colaborador I-10, entrevista, 21/06/2013).

A pesar de que los ministerios de salud de la región no intervinieron en cuanto al proceder del ICP, el personal tenía la presión de responder a estas entidades que aumentaron los pedidos de suero; ahí se empezó a identificar la fuente de los problemas. Los microorganismos eran actantes que no se habían visualizado $y$ eran los responsables de sabotear la red sociotécnica para la producción de sueros.

Para evitar toda conexión con estos actantes no invitados a participar, se tuvo que involucrar a otros nuevos: se comenzaron a realizar las pruebas de control de calidad de sangre y plasma, se levantaron registros de cada animal y de cada sangría, se llevaron a cabo procesos de desinfección de los caballos en la zona en la que se hacía la punción, además, se estableció que cada animal solo debía recibir una única punción con el catéter y se identificó el lugar idóneo y la forma correcta de hacerlo (la aguja debía introducirse de forma limpia, rápida y precisa en la yugular para evitar contaminación).

Asimismo, las bolsas para depositar la sangre se cambiaron y se comenzó a utilizar bolsas que permitían un mejor manejo y menor manipulación de la sangre para evitar al máximo su contaminación. Se empezó a trabajar con la inmunización, las dosis de veneno con las que se inoculaban los caballos fueron midiéndose y ajustándose; incluso se diseñaron esquemas de inmunización, porque existía la creencia de que entre más veneno, más anticuerpos producía el animal, pero en realidad se les provocaba lesiones graves $y$ dolorosas que podían ser fatales.

El personal que se dedicaba a la producción de suero tenía una escolaridad baja, no había un solo profesional, excepto por el veterinario, entonces hubo que reeducar a los trabajadores para que aprendieran los nuevos procesos y cambiar mentalidades, derribar mitos e incluso descartar creencias. Uno de los puntos clave del proceso era que el equipo contaba con gran compromiso y motivación para encargarse de la labor encomendada. El esquema 1 ilustra los cambios y las principales conexiones entre actantes que se involucraron en la red de producción de sueros antiofídicos para su mejoramiento: 
ESQUEMA 1

\section{NUEVOS ACTANTES Y SUS CONEXIONES PARA MEJORAR LA RESPUESTA AL PROBLEMA} DE LA PRODUCCIÓN DE SUEROS ANTIOFÍDICOS

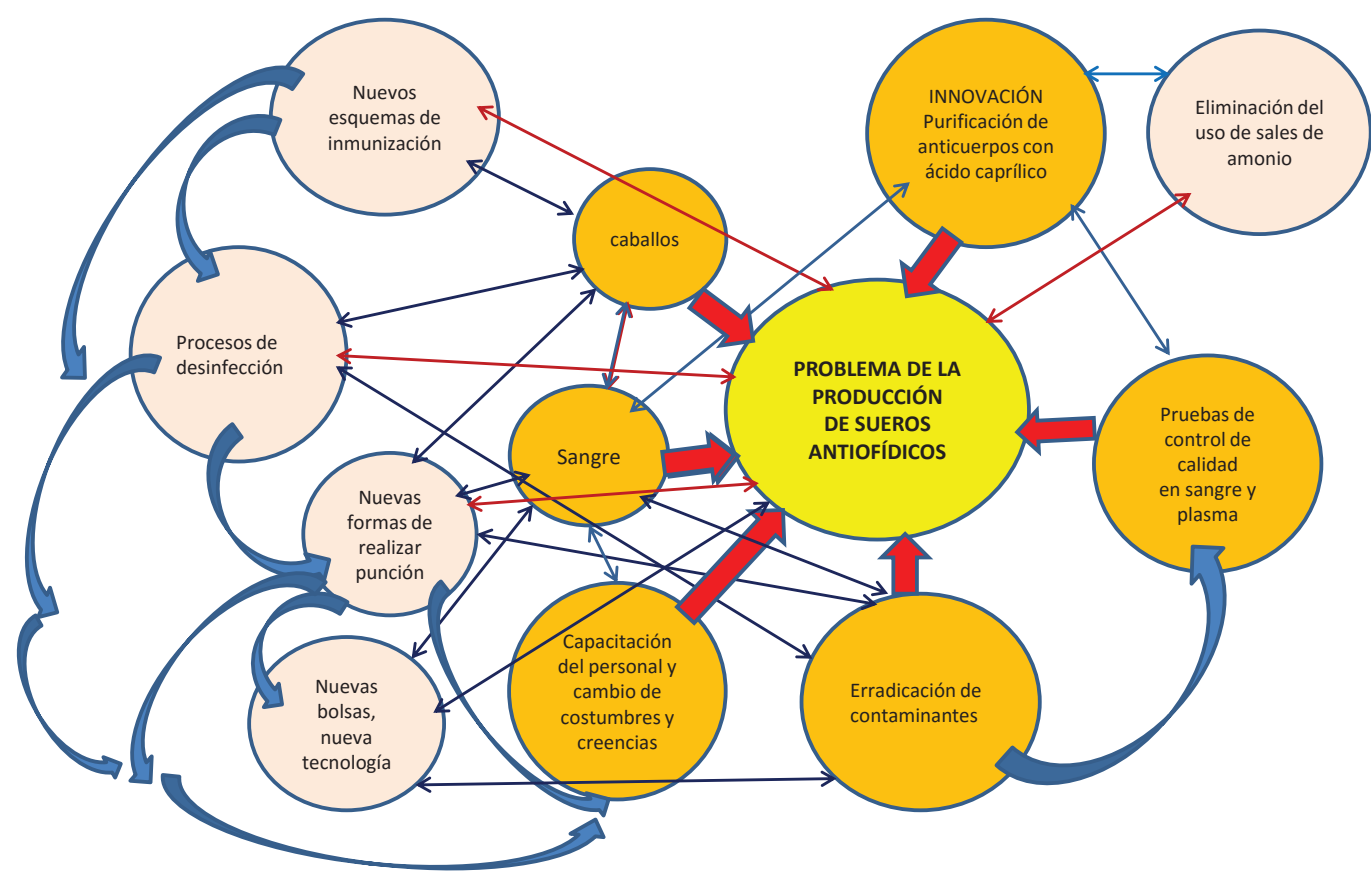

Fuente: Elaboración propia.

El círculo amarillo representa el problema central en torno al cual se agrupan los demás actantes y las flechas rojas muestran la conexión directa de todos con el problema. Las flechas azules y celestes ilustran las conexiones entre actantes, los importantes cambios que se introdujeron para la manipulación de los caballos, el desarrollo de protocolos para los esquemas de inmunización, procesos de desinfección y nuevos procedimientos para realizar la punción a los caballos y extraer su sangre con anticuerpos, la cual se depositó en nuevas bolsas manufacturadas con nueva tecnología para garantizar que no se contaminara su contenido, con el fin de erradicar los contaminantes que impedían el éxito en producción de lotes de suero. Se instauraron pruebas de control de calidad en cada uno de los procesos para comprobar la pureza de la sangre, las inmunoglobulinas y el suero.

La innovación del ácido caprílico para precipitar las inmunoglobulinas de la sangre de los caballos en vez de sales de amonio, representó un salto cuantitativo y cualitativo en la producción de sueros antiofídicos y todas estas transformaciones contribuyeron a la obtención del $100 \%$ de los lotes con una mejor calidad y pureza, en una cuarta parte del tiempo y a muy bajo costo:

Lo que va a suceder es que hacia finales de la década de 1990 y principios de la década siguiente, el Instituto está produciendo una cantidad de sueros cercana a los 60000 frascos, que le permite cubrir prácticamente la totalidad de la demanda en Centroamérica (colaborador I-10, entrevista, 21/06/2013). 
Paralelamente, mientras se desarrollaba el debate interno y se iniciaba el mejoramiento en los procesos de producción y el diseño de protocolos, la dirección enfrentaba otro obstáculo en el ámbito de la administración universitaria, pues en ese momento la Universidad no tenía una sola empresa y no había normativa ni mecanismos que facilitaran la exportación, mucho menos de un producto farmacéutico. La venta y el cobro del suero a los clientes del IсP era una labor ardua y desgastante, la cual era efectuada por el director, pero esto no era efectivo.

A partir de esto, se inició el trabajo con entidades distribuidoras que se encargaron de la entrega de los pedidos y la recaudación de dinero. Al interior de la Universidad no se sabía cómo actuar con estos nuevos procesos, no había normativa ni procedimientos establecidos, sino desconocimiento y prejuicio. La administración no apoyaba los esfuerzos del IcP, impidiendo cumplir con los compromisos adquiridos, debido a la contradicción de que una dependencia universitaria funcionara con una lógica empresarial.

Por otro lado, la pérdida constante de lotes de suero no indicaba avances. Durante aproximadamente tres años se luchó contra los problemas de producción, sin poder entregar un solo pedido a tiempo a los países vecinos. Para mejorar, había que replantear varios aspectos y tomar decisiones, pues de otra forma el reconocimiento y la responsabilidad otorgada al ICP y a la ucR estaban en un gran riesgo de fracasar. Además vendría un nuevo reto que debían afrontar y que no podían eludir.

\section{LA CONFORMACIÓN DE LA RED SOCIOTÉCNICA PARA LA CREACIÓN DEL SUERO PAN-AFRICANO}

En ese escenario, el Dr. Rojas recibió una invitación para que el ICP participara en una reunión convocada por la Organización Mundial de la Salud (oms), que tuvo lugar en Inglaterra en febrero del año 2001, por lo cual mandó como representante al Dr. José María Gutiérrez. En este evento, participaron tanto personas de países afectados por un alto índice de accidentes ofídicos, como distintas instituciones públicas y privadas productoras de suero de distintos países del mundo.

En la reunión, cada uno dio su visión de cómo eran las cosas en cada país, se hizo como un diagnóstico de la situación de sueros antiofídicos. Un tema que tuvo mucho peso, fue la situación de falta de sueros antiofídicos en África Subsahariana, fue un punto muy fuerte de la reunión, hubo gente de África que lo planteó con mucha vehemencia (José María Gutiérrez, entrevista, 02/12/2013).

Esta región tiene serpientes muy venenosas que provocan miles de accidentes y muertes cada año; sin embargo, ninguno de estos países produce sueros en la actualidad. Solo en Sudáfrica hay una empresa que produce una pequeña cantidad de antídotos para países del sur de África. El Dr. Gutiérrez consideró que para asumir este reto debían involucrarse varios productores de sueros, por lo cual partiendo de sus valores y el carácter social del ıсp, hizo la siguiente propuesta:

... una posible solución parcial o enfrentamiento parcial a ese problema tan grave, era que algunos laboratorios productores nos comprometiéramos con una cuota de suero para África y que si varios laboratorios, aunque la cuota fuera pequeña, si cada uno pusiéramos de nuestra parte, se podría contribuir de una manera significativa a alivianar este problema de falta de sueros antiofídicos (José María Gutiérrez, entrevista, 02/12/2013).

La iniciativa tuvo acogida en varios de los participantes y el Dr. Gutiérrez se comprometió por parte del ICP a desarrollar un suero para los países de África Subsahariana y de una vez, planteó la posibilidad de realizar esta iniciativa asociándose en una red con el grupo conformado por la Universidad de Oxford y la Escuela de Medicina Tropical de la Universidad de Liverpool y la empresa inglesa MicroPharm, quienes se habían aliado con el Ministerio Federal de Salud de Nigeria (MFSN). Este grupo venía 
trabajando desde los años 80 en el desarrollo de un suero que ya se estaba utilizando en pacientes humanos, pero no daban abasto para la atención de miles de víctimas que hay cada año en esa región del mundo:

... tenían una organización que se llama Echi TAb Study Group. "Echi" viene porque la serpiente más importante de esa zona se llama Echis ocellatus ${ }^{7}$ y "TAb" porque la empresa se llama Therapeutics Anti-bodys, ellos habían propuesto Echi TAb Study Group y como el suero que habían hecho los ingleses dio buenos resultados, aparentemente ese nombre EchiTAb era un nombre como que suena bien en Nigeria, etcétera. Así el Instituto comunicó que estaba de acuerdo en formar parte en ese proyecto
(José María Gutiérrez, entrevista, 02/12/2013).

Por su parte, se comprometieron con este reto los representantes de la empresa Bioclon de México, del Instituto Butantan de Brasil y del Instituto Nacional de Salud de Colombia, que tenía problemas organizativos $y$ de producción. La OMS fue en ese momento un actante central que propició el escenario en la creación de una red mundial para la producción de sueros antiofídicos, tuvo el poder para convocar a las entidades productoras de sueros y también involucró a representantes de los países que sufrían por la ausencia de antivenenos para atender a víctimas de mordeduras de serpientes venenosas. El Dr. Gutiérrez aprovechó el espacio, motivó la participación de otros actantes en el reto y solicitó unirse a la red constituida por el Echi TAb Study Group (esquema 2).

ESQUEMA 2

\section{INICIO DE LA RED SOCIOTÉCNICA PARA LA PRODUCCIÓN DE SUEROS ANTIOFÍDICOS PARA PAÍSES DE ÁFRICA SUBSAHARIANA}

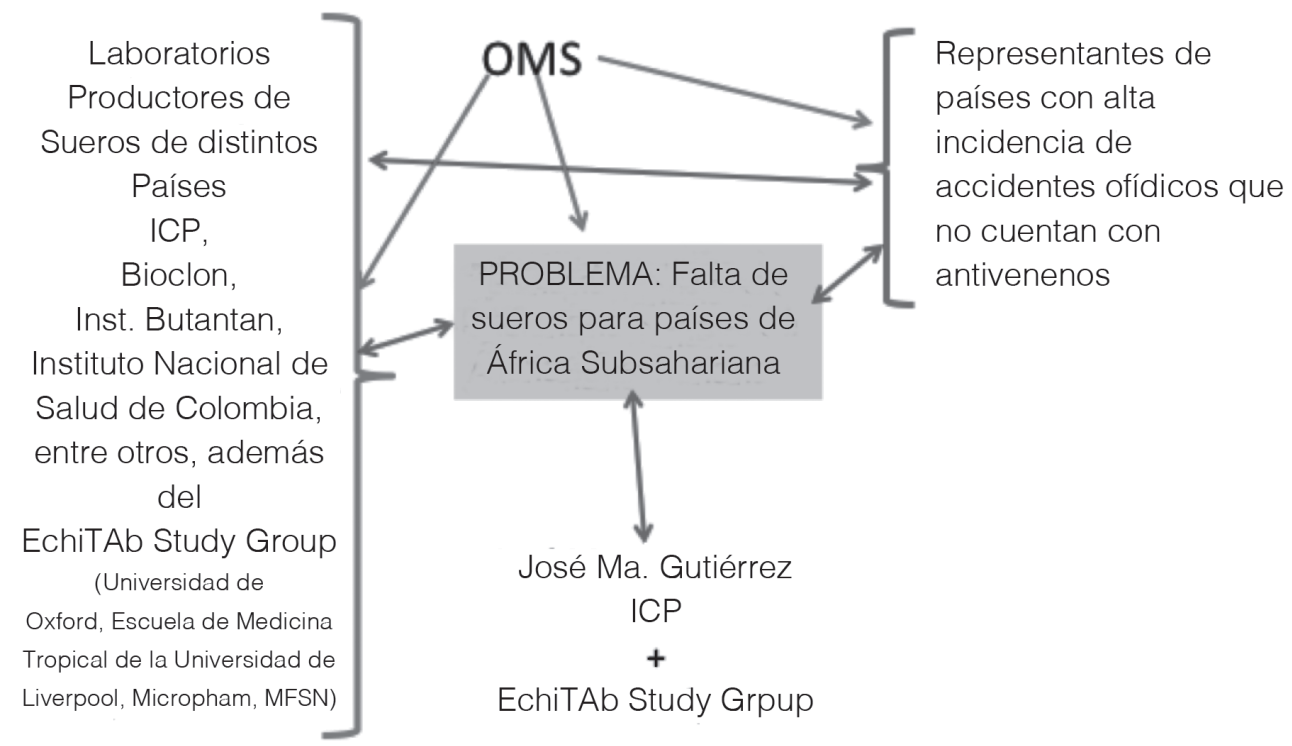

Fuente: Elaboración propia.

$7 \quad$ Conocida comúnmente como víbora de alfombra. 
$\mathrm{Al}$ asociarse el ICP con este grupo, se conformó una nueva red - la oms no participó- en la cual estos actantes asumieron su rol, su compromiso, pactaron alianzas y pusieron en circulación varios actantes no humanos en torno al problema del desarrollo de una nueva variedad de suero para países de África Subsahariana. Como puede apreciarse en el esquema 2, el MFSN se comprometió a colectar las serpientes y enviarlas a la Escuela de Medicina Tropical en Liverpool, esta a su vez asumió la responsabilidad de extraer el veneno de las serpientes y enviarlo al ICP; el Dr. David Warrell de la Universidad de Oxford aportaría con su vasto conocimiento en serpientes africanas, sus venenos $y$ sus efectos; la empresa MicroPharm colaboraría con su experiencia en la elaboración del suero EchiTAb, así como en la realización del estudio preclínico para probarlo. Por su parte, el ICP se comprometió a utilizar los aportes de los demás actantes $y$ desarrollar una nueva variedad de suero polivalente para combatir envenenamientos por mordeduras de distintas serpientes africanas, a partir de la experiencia en la elaboración de sueros, su personal calificado, su infraestructura, su tecnología, así como, el diseño de experimentos y pruebas. El esquema 3 muestra los actantes que inicialmente conformaron la red, sus conexiones y los actantes no humanos que pusieron en circulación, para dar una respuesta al problema de la falta de sueros antiofídicos para África Subsahariana:

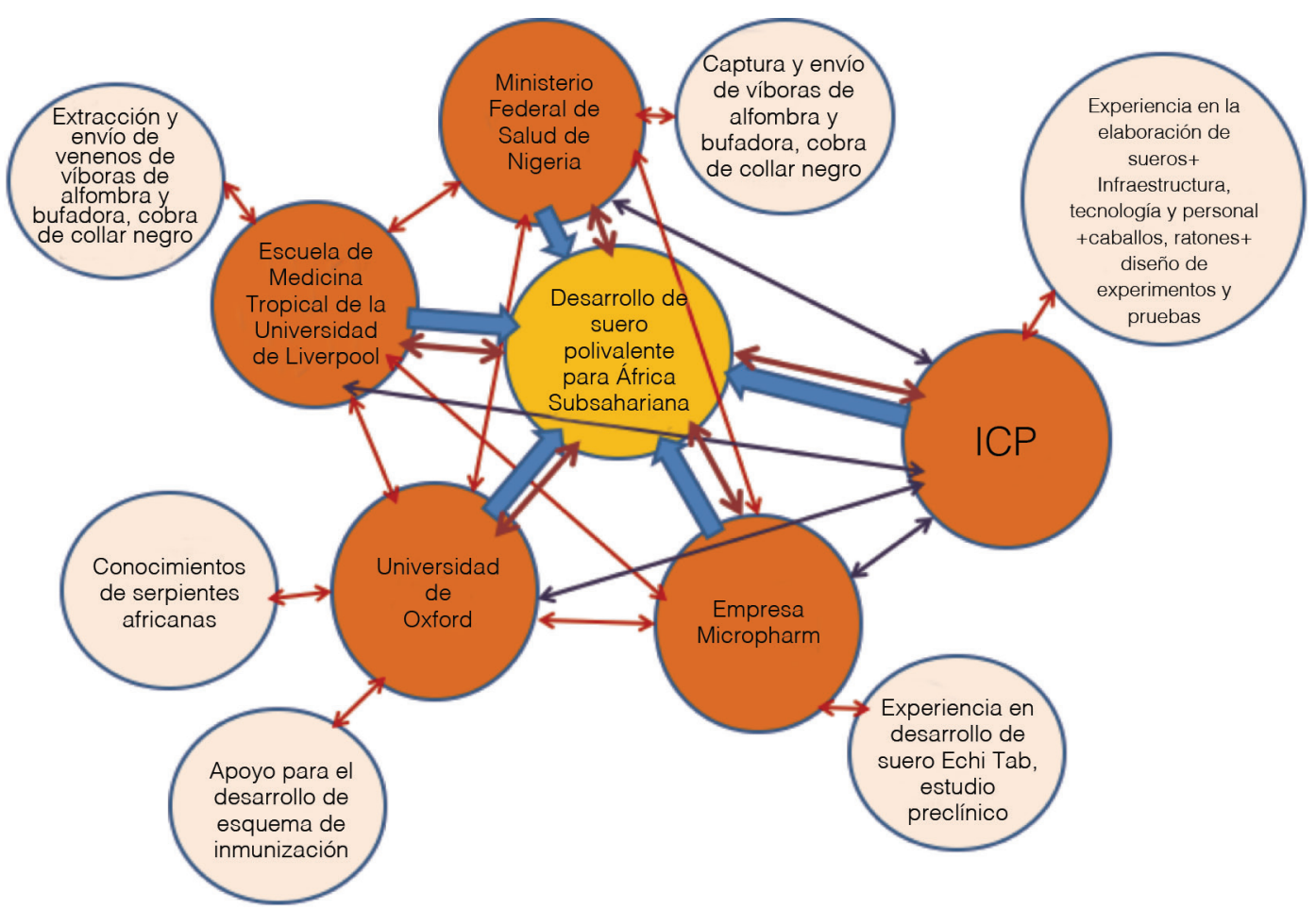

Fuente: Elaboración propia. 
El Dr. Rojas respalda el compromiso adquirido por del Dr. Gutiérrez, aunado a que los problemas de producción comenzaron a solucionarse:

Un día purgamos el último demonio del proceso, hicimos un lote bien, luego otro bien y otro más. Consecuentemente todos los lotes empezaron a salir bien, en un momento en que de necesitar dos lotes había que hacer diez, ahora teníamos los diez sin problema de pirogénesis. Esa promesa que José María hizo en la reunión de la oms, dos años antes hubiese sido imposible cumplirla porque simple y sencillamente no teníamos como, ni siquiera solo para Costa Rica, ni siquiera para Centroamérica, menos hacer suero para Nigeria (Guillermo León, entrevista, 20/11/2013).

Con un mejor control de todos los procesos de la producción, se inició el desarrollo del suero para los países de África Subsahariana, conformando una red que involucró a actantes de tres continentes.

Tenemos mejores títulos ${ }^{8}$ de caballos, con una planta que resuelve los lotes en menos tiempo, entonces se subió la eficiencia. De ahí en algún momento dijimos ¿qué hacemos con todo este tiempo de planta?, ¿podemos hacer suero para Centroamérica? Probemos. ¿Podemos hacer suero para otros lados?-Probemos, porque ahora entran en la planta.

No obstante, era muy diferente producir sueros con venenos de serpientes conocidas que de serpientes lejanas y de otras especies con las que nunca se había trabajado:

Lo primero que se hizo fue que los personeros en Nigeria e Inglaterra, puesto que cuentan con los serpentarios, nos mandaran veneno de tres especies de Nigeria, la Echis ocellatus; una víbora bufadora ${ }^{9}$

8 Respuesta inmune.

$9 \quad$ Bitis arietans. $y$ una serpiente de la familia Elapidae, de la familia de las corales, una cobra ${ }^{10}$. Empezamos a experimentar, seleccionamos unos caballos, les aplicamos el veneno $y$ en fin, varios funcionarios del Instituto participamos con una actitud muy positiva (José María Gutiérrez, entrevista, 02/12/2013).

En el ICP se conformó una red sociotécnica en la que participaron la División Académica y la División Industrial. El Dr. Gutiérrez hizo pruebas, análisis y experimentos con los venenos de las serpientes africanas, obteniendo información de vital relevancia sobre la composición molecular de los venenos utilizada en la Sección de Producción, aunque en principio eran diferentes a los de las serpientes de Costa Rica, tenían una química similar. Este dato fue fundamental para el diseño del esquema de inmunización, como se explicará más adelante.

El desarrollo simultáneo de una nueva variedad de suero junto al que se elaboraba para los países centroamericanos, requería más recursos para la producción y el aumento del número de caballos; no obstante, el terreno del ICP estaba saturado (se tenían 40 animales en una hectárea siendo lo ideal un caballo por hectárea). Como un mecanismo de apoyo, dos funcionarios del área de producción que contaban con fincas propias, decidieron ofrecer el terreno para la creciente producción, creando una empresa llamada MIMESOL y proseguir a inmunizar los caballos con los venenos de las serpientes africanas. Esta acción generó una nueva controversia al interior del ICP:

... ellos dieron el paso [los compañeros de mimesol] también al poner en juego sus fincas $y$ dinero para la compra de caballos y demás ...estoy convencido, de la buena intención de ayudarle al instituto, aquí se vio mal porque parecía como que ellos eran juez y parte, de que se estaban atiborrando de dinero...(colaboradora I-10, entrevista, 20/11/2013).

$10 \quad$ Naja nigricollis conocida comúnmente como cobra escupidora de cuello negro. 
Los registros contables muestran que la empresa no tuvo mayores ganancias, MIMESOL solo mantuvo a cuatro caballos con los que se probó un esquema de inmunización similar al que se usaba para elaborar el suero polivalente ${ }^{11}$. Las principales preocupaciones eran el fracaso $y$ la muerte de los caballos; no obstante obtuvieron resultados positivos. Es importante señalar que la alianza estratégica que el Dr. Gutiérrez motivó desde el inicio con las entidades inglesas mencionadas fue crucial, pues el Dr. David Warrell de la Universidad de Oxford, con su amplio conocimiento sobre serpientes africanas, apoyó el diseño del esquema $y$ aportó pautas para la inoculación con los distintos venenos.

Optamos por lo simple, hacer lo que se venía haciendo con el polivalente; ese era nuestro punto de partida con el de África $y$ desde ése momento el suero empezó a salir muy bien. Después de eso lo hemos venido mejorando, hemos venido... cada vez que inmunizamos caballos son experimentos que duran dos meses, una inyectada $y$ dos meses después se tiene derecho a otra inyectada, y otra (colaboradora $\mathrm{I}-10$, entrevista, 28/03/2014).

La experiencia y el conocimiento acumulados, principalmente las técnicas y protocolos desarrollados entre 1998-2001, permitieron aplicar tecnología conocida y probada con venenos desconocidos y muy diferentes. Dadas las circunstancias, el éxito del esquema según las percepciones de las personas involucradas, fue considerado una casualidad, ya que el mismo esquema de inmunización funcionó con los venenos de serpientes africanas.

11 Según Gutiérrez: "el suero polivalente se produce mediante la inmunización activa de caballos adultos sanos con una mezcla de partes iguales de los venenos de Bothrops asper (terciopelo), Crotalus simus (cascabel) y Lachesis stenophrys (cascabela muda o matabuey)". El caballo desarrolla anticuerpos, se le extrae sangre, la cual es purificada mediante procesos de laboratorio para separar del plasma los anticuerpos y proteínas que constituyen la materia prima para la elaboración del suero (c.p. dic., 2011).
Esos caballos pudimos haberlos inmunizado, aproximadamente unos tres meses, $y$ el resultado era incierto, al final salió bien por un tremendo golpe de suerte, de que el esquema que estábamos utilizando para nuestros caballos funcionara con esos otros venenos (Guillermo León, entrevista, 20/11/2013).

Esta nueva variedad de suero logró desarrollarse debido a la vinculación del área de producción con el área académica del ICP y la estructura administrativa universitaria, además de las entidades académicas británicas (Universidad de Oxford y Universidad de Liverpool), la empresa MicroPharm, el sector salud del gobierno de Nigeria, asimismo, al dominio de la técnica que había desarrollado el Dr. León y su equipo en la División Industrial. Es importante señalar que todos los cambios en la producción de sueros le valieron al Dr. León, el Premio Nacional de Tecnología ${ }^{12}$ en el año 2009.

En el 2011, se otorga al Instituto Clodomiro Picado, el Premio "Dr. Lee Jong-Wook Memorial Prize" de la Organización Mundial de la Salud, por contribuir a un problema de salud poco atendido, como son los accidentes ofídicos. Las personas involucradas consideran que el papel de cada uno de los actores fue fundamental parar desarrollar este producto:

... fue una coyuntura de muchas cosas; primero la reunión de la oms, que José María pudiera asistir, que no se arruga$\mathrm{ra}^{13}$ a la hora de dar el paso adelante $y$ trajera la oportunidad para el instituto. $\mathrm{El}$ instituto que por otro lado hubiera podido resolver sus problemas de producción y sentir de alguna manera, incluso una holgura en la producción para esos meses, y el esfuerzo de compañeros que quisieron apoyar metiendo animales para hacer la experiencia. Solamente la participación de José María en la reunión

12 Este premio lo otorga cada año el Ministerio de Ciencia y Tecnología del gobierno de Costa Rica.

13 En lenguaje popular quiere decir que no se arriesga y no acepta la oportunidad. 
sin los compañeros que nos permitieran manejar caballos [en sus fincas], simplemente no hubiéramos podido hacerlo. Solamente los caballos y nosotros desarrollando músculo productivo sin que José María vaya a la reunión, simplemente no nos percatamos de la oportunidad y no lo hacemos (Guillermo León, entrevista, 28/03/2014).

En medio de la organización para producir el suero, las autoridades de salud de Nigeria hicieron una solicitud expresa al ICP de mantener el nombre EchiTab del suero, el producido en Inglaterra se denomina EquiTab-G, pero el del ICP es un suero polivalente que permite atender envenenamientos producidos por muchas serpientes diferentes, así que se denominó EchiTab-plus-ICP:

... pero lo que acordamos fue - en realidad estas personas son nuestros clientes, ellos son los que saben cuál es el escenario y saben cuál es el nombre más conveniente- Les dijimos que estábamos de acuerdo con EchiTAb-plus pero siempre y cuando le pongamos al final ICP ( José María Gutiérrez, entrevista, 02/12/2014).

En este caso, el grupo social que impulsó de forma vehemente el desarrollo de una variedad de suero para África Subsahariana influyó también en el mismo nombre del producto del cual sería receptor, aunque en términos comunes el suero es llamado de una forma diferente, gracias a su capacidad neutralizante de una importante variedad de envenenamientos producidos por distintas serpientes africanas, Panafricam antivenom en inglés. En el esquema 4, se muestran las conexiones entre todos los actantes vinculados con el problema del desarrollo del suero polivalente para países de África Subsaharina.

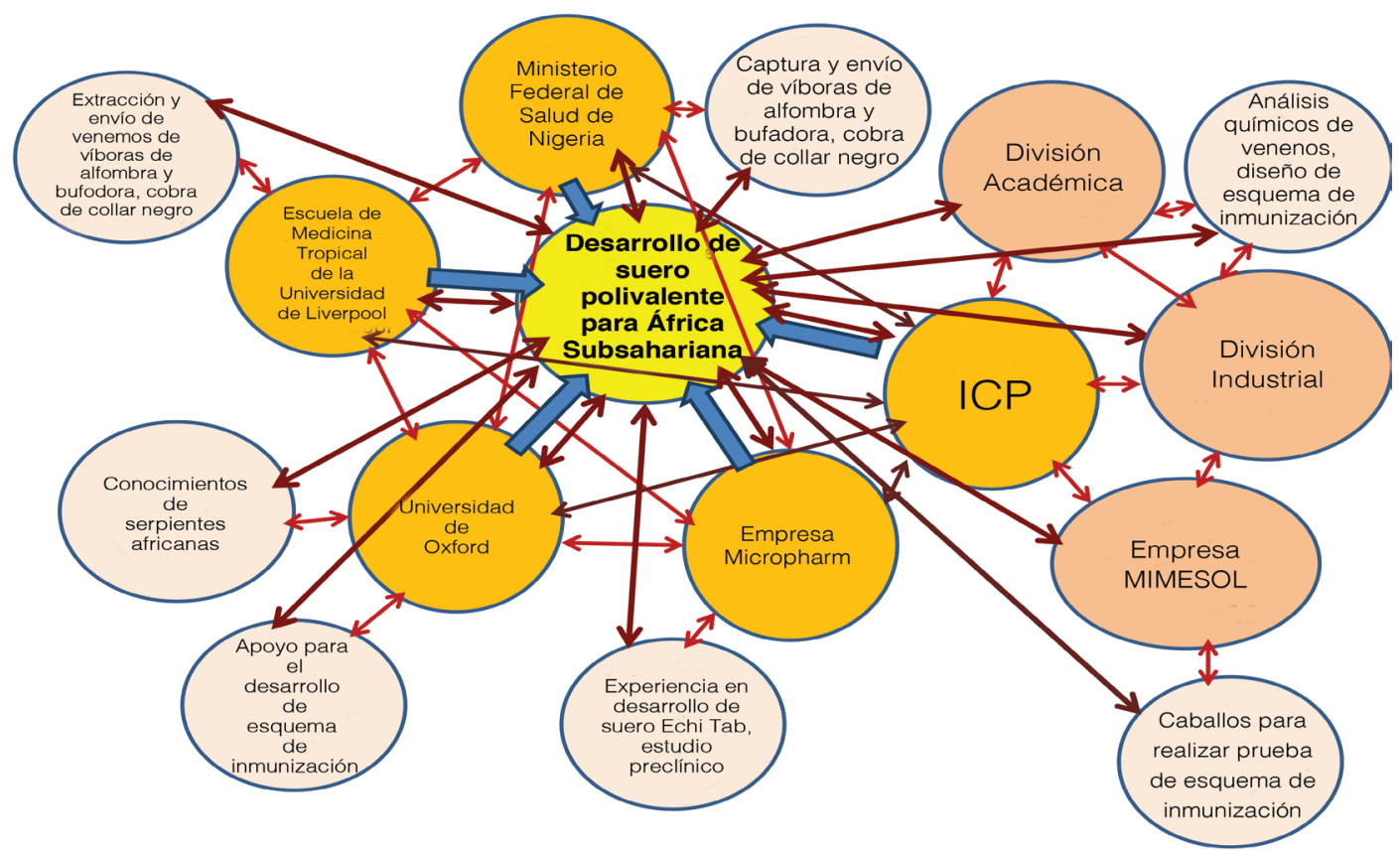

Fuente: Elaboración propia. 
El cumplimiento de roles de todos los actantes involucrados en la red, permitió diseñar un exitoso esquema de inmunización que dio como resultado la nueva variedad de suero. Además de los actantes involucrados al inicio, ingresó a la red un nuevo actante, la empresa Mimesol, que aportó cuatro caballos que fueron inmunizados con los venenos de las serpientes africanas y el buen resultado demostró la efectividad del diseño. Las flechas moradas muestran la conexión de todos los actantes con el problema $y$ las flechas rojas $y$ azules, las conexiones entre actantes.

Además de aumentar la población de caballos para inmunizar, producir este suero implicó muchos cambios. Se hizo necesario contratar personal profesional que viniera a apoyar los retos y el crecimiento que implicaba este desarrollo, también crear el andamiaje para darle un carácter industrial y empresarial, sin perder de vista la función social de producir los antivenenos para salvar vidas, con la mejor calidad y al más bajo costo posible.

Se alquiló una pequeña finca cercana, porque era urgente sacar los caballos de las instalaciones del ICP en Dulce Nombre de Coronado. Luego, se alquiló una finca de 40 hectáreas en Cascajal de Coronado, que posteriormente fue adquirida por la Universidad para ubicar a la creciente población de caballos. Así, con los primeros cuatro caballos inmunizados bajo el cuidado de MIMESOL se elaboraron las primeras dosis de suero panafricano y se realizó el ensayo clínico para probar un suero antiofídico, con 400 pacientes. El ICP coordinó el ensayo clínico con las autoridades de salud de Nigeria, quienes se hicieron cargo por completo de su ejecución:

En ese ensayo clínico se compararon el EchiTAb-G, que ya estaba bien comprobado en Nigeria, y el nuestro que era nuevo el EchiTAb-plus-ICP; los ensayos clínicos tienen diferentes tipos de diseño, el de ese es un diseño que se llama Non Inferiorty Trial, un ensayo de no inferioridad. La hipótesis es que el producto nuevo que se va a probar no es inferior al producto existente, así es como se plantea esos proyectos. El proyecto duró varios años, nosotros no nos metimos en nada en ese proyecto, nada más donamos los sueros (...) en el año 2009 nos dijeron -nosotros queremos ir a Costa Rica, el proyecto salió muy bien y queremos ir a presentarles los resultados del proyecto- entonces en 2009 fue un año memorable para nosotros, llegaron tres nigerianos, el Dr. Nasidi, director del proyecto; el Dr. Habib que es el clínico encargado de ese proyecto (José María Gutiérrez, entrevista, 02/12/2014).

La Universidad de Oxford y la empresa Micropharm no participaron en el estudio clínico sino que este se realizó directamente por el MFSN en coordinación con el IcP, que siguió recibiendo el suministro de venenos por parte de la Escuela de Medicina Tropical de la Universidad de Liverpool, materia indispensable para la elaboración del suero EchiTAb-plus-ıcp que debía enviar a Nigeria.

En su visita a las instalaciones del ICP en el año 2009, el Dr. Adulrazaq Habib ${ }^{14}$ indicó que en su país ocurren cerca de 150000 accidentes ofídicos al año y sin el tratamiento apropiado, el $20 \%$ de las personas afectadas puede morir. Gracias al uso del suero EchiTAbplus-ICP en ese momento, las muertes se habían reducido a cero. El ICP suministró de forma gratuita el suero al Ministerio Federal de Salud de Nigeria por varios años $y$ de igual forma la Escuela de Medicina Tropical de la Universidad de Liverpool, Inglaterra, aportó gratuitamente los venenos de las serpientes, que son la materia prima con la que inicia el proceso de producción de suero.

Cuando el suero pasó la prueba del estudio clínico, correspondió registrar este nuevo suero en el Ministerio de Salud de Costa Rica $y$ en Nigeria, lo cual conllevo un tiempo hasta que finalizó el proceso de trámites burocráticos en el año 2010. En el año 2013, el Dr. Habib publicó un estudio sobre la efectividad de los dos sueros, el EchiTAb-G y el EchiTAb-plus-ICP, en

14 Información consultada en http://www.ucr.ac.cr/ noticias/2009/03/25/ucr-produce-suero-antiofidico-para-nigeria/imprimir.html 
el tratamiento de víctimas de accidentes ofídicos en Nigeria, demostrando que el $83 \%$ de las personas tratadas con el antiveneno desarrollado en el ICP se recuperaron completamente al término de dos días de haber recibido el tratamiento, contra un $76 \%$ de víctimas tratadas con el suero diseñado por la empresa británica. Asimismo, el suero costarricense ha demostrado su capacidad neutralizante contra el veneno de distintas serpientes africanas, tanto víboras como cobras (Habib, 2013). Estos resultados causan orgullo y satisfacción en el personal del ICP que participó en la elaboración del suero.

En el año 2011, el ICP hizo una inversión en caballos, terrenos, infraestructura, equipamiento y contratación de nuevo personal, pues además de la donación de venenos, no recibieron ningún aporte de instituciones externas. Por otra parte, al interno del Instituto, la discusión sirvió para tomar la decisión de destinar recursos propios en este proyecto por su carácter humanitario. Así se facilitó todo el suero que permitió hacer los ensayos clínicos y se continuó entregando entre 7000 y 9000 viales de suero por año a Nigeria.

La red que inició en 2001, con el espacio en la reunión que propició la oMs - aunque la búsqueda para la solución al problema de los accidentes ofídicos en los países de África Subsahariana se logró con la elaboración del suero EchiTAb-plus-ICP - continúa la producción de suero hasta el presente, aunque el papel de algunos actores ha variado $y$ algunos estuvieron solo durante un momento determinado, pues su rol había sido cumplido.

Un dato interesante a partir de las percepciones de las personas entrevistadas, es que pese a la diversidad de actantes que se han involucrado en el desarrollo de este suero, no hubo conflictos en el proceso y las relaciones entre las partes involucradas transcurrieron en un ambiente de cordialidad, donde cada quien respondió a las expectativas del grupo según su función en la red y el aporte que debía realizar para lograr el objetivo común. El Dr. Gutiérrez fue un actante central en la red y posteriormente, el Dr. León con su equipo de la División
Industrial, tuvieron también la centralidad y salvo las controversias sobre ciencia versus tecnología o aporte humanitario versus negocio privado, los participantes en la red no tuvieron ningún conflicto, como lo explica esta persona: “... siempre había una sintonía filosófica de a dónde se quería ir y por qué ..." (colaboradora I-09, entrevista, 28/03/2014).

\section{CONCLUSIONES}

Once años después de iniciar el proyecto para la producción del suero panafricano, el Ministerio de Salud de Nigeria inició la compra de suero al ICP en el año 2012, pues con solo dos frascos de suero se logra tratar de manera satisfactoria a los pacientes con excelentes resultados, debido a la pureza del suero, según manifestó el Dr. Nasidi al Dr. Gutiérrez y de igual forma, el Dr. Habib (2013) lo señala en su artículo. También otros países como Burkina Fasso, Malí y Ghana están comprando el suero lo cual representa un importante logro para el personal del Instituto, puesto que la organización Médicos Sin Fronteras lo aprobó y compró una pequeña cantidad de frascos. Para quienes han desarrollado el medicamento, este hecho representa un reconocimiento tácito a su esfuerzo, también a la calidad de su producto, comprobado por esta institución, la cual tiene altos estándares de calidad para definir los medicamentos adquiridos.

La Escuela de Medicina Tropical de la Universidad de Liverpool ahora le vende los venenos al ICP, que aunque con un precio elevado, tienen buena calidad y rendimiento. Asimismo, un nuevo actor se ha sumado a la red, un comerciante costarricense de origen taiwanés, quien es intermediario promocionando el suero en los países africanos donde puede ser utilizado. La producción de EchiTAb-plus-ICP va en aumento $y$ por eso hay cambios necesarios; a partir de la compra de suero por parte de Nigeria, el ICP también inició la compra de veneno a la Liverpool Royal School of Tropical Medicine, por lo que ahora la relación es académica y 
comercial, aunque los nigerianos continúan enviado las serpientes a Inglaterra.

El suero no fue el único producto de este proceso, sino que se han generado varias publicaciones que involucran al personal del icp de la División Académica y la División Industrial, académicos de la Universidad de Liverpool y de la Universidad de Oxford, personal de salud de Nigeria y de la empresa MicroPharm; incluso, una primera publicación involucró tanto al personal del Instituto de Salud de Colombia como al Instituto de Biomedicina de Valencia, España, en los últimos años, en la persona del Dr. Juan José Calvete. Paralelamente a la investigación aplicada para el desarrollo del suero, se llevó a cabo un componente de investigación básica para la caracterización de los venenos, las proteínas tóxicas que contienen y sus efectos; debido a esto el ICP incursionó en la investigación sobre proteómica, de modo que las publicaciones dan cuenta del conocimiento básico que se generó en el proceso:

$\diamond \quad$ Laing, G.D., Renjifo, J.M., Ruiz, F., Harrison, R.A., Nasidi, A., Gutiérrez, J.M., Rowley, P.D., Warrell, D.A. \& Theakston, R.D.G. (2003) A new Pan African polyspecific antivenom developed in response to the antivenom crisis in Africa. Toxicon 42, 35-41.

$\diamond \quad$ Gutierrez JM, Rojas E, Quesada L, Leon G, Nunez J, Laing GD, Sasa M, Renjifo JM, Nasidi A, Warrell DA, Theakston RDG and Rojas G (2005). Pan-African polyspecific antivenom produced by caprylic acid purification of horse IgG: an alternative to the antivenom crisis in Africa. Transactions of the Royal Society of Tropical Medicine and Hygiene 99: 468-475.

$\diamond \quad$ Segura, A., Villalta, M., Herrera, M., León, G., Harrison, R., Durfa, N., Nasidi, A., Calvete, J.J., Theakston, R.D.G., Warrell, D.A. \& Gutiérrez, J.M. (2010)
Preclinical assessment of the efficacy of a new antivenom (EchiTAb-Plus-ICP $\AA$ ) for the treatment of viper envenoming in sub-Saharan Africa. Toxicon 55, 369-374.

$\diamond \quad$ Calvete, J.J., Cid, P., Sanz, L., Segura, A., Villalta, M., Herrera, M., León, G., Harrison, R., Durfa, N., Nasidi, A., Theakston, R.D.G., Warrell, D.A. \& Gutiérrez, J.M. (2010) Antivenomic assessment of the immunological reactivity of EchiTAb-Plus-icp, an antivenom for the treatment of snakebite envenoming in sub-Saharan Africa. American Journal of Tropical Medicine and Hygiene 82, 1194-1201.

$\diamond \quad$ Petras, D., Sanz, L., Segura, A., Herrera, M., Villalta, M., Solano, D., Vargas, M., Leon, G., Warrell, D.A., Theakston, R.D.G., Harrison, R.A., Durfa, N., Nasidi, A., Gutierrez, J-M., Calvete, J.J. (2011) Snake venomics of the African spitting cobras: Toxin composition and assessment of congeneric cross-reactivity of the Pan-African EchiTAb-Plus-ICP antivenom by antivenomics and neutralization approaches. Journal of Proteomic Research 10:1266-1280.

$\diamond \quad$ Méndez, I., Gutiérrez, J.M., Angulo, Y., Calvete, J.J., \& Lomonte, B. (2011) Comparative study of the cytolytic activity of snake venoms from African spitting cobras (Naja spp., Elapidae) and its neutralization by a polyspecific antivenom. Toxicon 58, 558-564.

El desarrollo de la nueva variedad de suero implica pensar de manera prospectiva, lo cual va a requerir de nuevas discusiones al interno del ICP, tomar nuevas decisiones y definir las estrategias a seguir, pues se prevé que Nigeria y otros países africanos aumentarán su demanda del suero EchiTab-plus-ICP. Ahora la red sociotécnica estabilizada para la producción puede apreciarse en el esquema 5. 


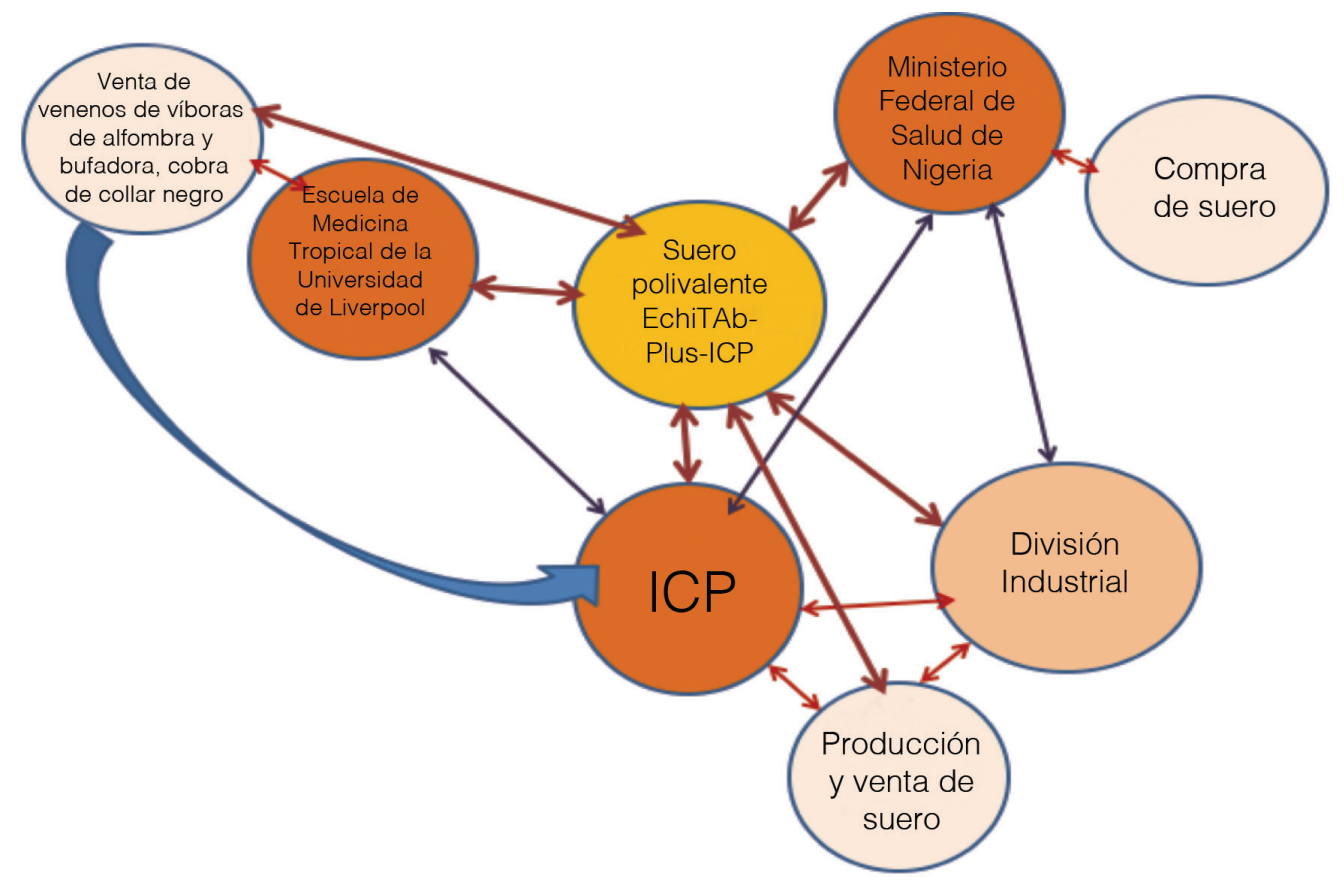

Fuente: Elaboración propia.

Una vez que el suero aprobó el ensayo clínico y el medicamento se registró en Costa Rica y Nigeria, actantes como la Universidad de Oxford y la empresa MicroPharm dejaron la red y el rol de los actantes remanentes se modificó. El mFsn comenzó a comprar el suero al ıсP que a su vez compró los venenos a la Universidad de Liverpool. La producción de suero sigue siendo el nodo central que motiva la conexión entre los actantes y mantiene la red unida y estabilizada.

BIBLIOGRAFÍA

\section{LIBROS}

Arellano, A. (1999). La producción social de los objetos técnicos agrícolas. Toluca, México: Universidad Autónoma del Estado de México.

Arellano, A. (2009). Antropología: Contribución de las etnografías de laboratorio al programa de la Antropología. En: Corona, L. (coord.). Enfoques de la Innovación ante la Sociedad de Conocimiento. México: Universidad Autónoma del Estado de México.

Arellano, A. (2015). Epistemología de la Antropología: conocimiento, técnica y hominización. México: Ediciones y Gráficos Eón.

Arellano, A. y Jensen, H. (2009). Mapeando las redes de investigación en Ciencias Básicas en la Universidad de Costa Rica. En: Viales, R.; Amador, J. y Solano, F. (eds.). Concepciones y representaciones de la Naturaleza y la Ciencia en América Latina. San José, Costa Rica: Universidad de Costa Rica, Vicerrectoría de Investigación, Cátedra Humboldt, Centro de Investigaciones Geofísicas, Programa de Estudios Sociales de la Ciencia, la Técnica y el Medio Ambiente. 
Jociles Rubio, M. I. (1992). Ciencia en acción. Cómo seguir a los científicos $e$ ingenieros a través de la sociedad. Madrid, España: Editorial Labor S.A.

Jociles, M. I. (2007). Nunca fuimos modernos. Ensayo de antropología simétrica. Buenos Aires, Argentina: Editorial Siglo XxI.

Jociles, M. I. (2008). Reemsamblar lo social. Una introducción a la teoría del actorred. Buenos Aires, Argentina: Ediciones Manantial SRL.

Ortega, C. (2013). Del laboratorio al campo: la historia inconclusa de la papa transgénica en México. En: Arellano, A.; Chauvet, M. y Viales, R. (coords.). Redes y estilos de investigación: Ciencia, Tecnología, Innovación y Sociedad en México y Costa Rica. México: Editorial Miguel Ángel Porrúa.

Ortega, C. y Arellano, A. (2010). Relaciones sociales y de genes. El primer vegetal transgénico mexicano. México: Editorial Miguel Ángel Porrúa.

Pinch, T. J. y Bijker, W. E. (2013). La construcción social de hechos y de artefactos: o acerca de cómo la sociología de la ciencia y la sociología de la tecnología pueden beneficiarse mutuamente. En Hernán, T. y Alfonso, B. (coords.). Actos, actores y artefactos. Sociología de la Tecnología. Argentina: Universidad Nacional de Quilmes Editorial.

\section{PUBLICACIONES PERIÓDICAS}

Arellano, A. (2003). La sociología de las ciencias $y$ de las técnicas de Bruno Latour y Michel Callon. Cuadernos Digitales: Publicación Electrónica en Historia, Archivística y Estudios Sociales, 8(23).

Carlson, W. B. y Gorman, M. E. (febrero, 1992). Socio-Technical Graphs and Cognitive Maps: A Response to Latour, Mauguin and Teil. Social Studies of Science, 22 (1).

Grau, M.; Íñiguez-Rueda, L. y Subirats, J. (noviembre, 2010). La perspectiva sociotécnica en el análisis de políticas públicas. Revista Psicología Política, 41: 61-80.
Gutiérrez, J. M. (2010). Biografía Dr. Clodomiro Picado Twight (1887-1944). Acta Médica Costarricense, 52(1).

Gutiérrez, J. M.; Lomonte, B.; Rojas, G.; Gené, J. A.; Chaves, F.; Estrada, R.; Alvarado, J.; Rojas, E. y Robles, A. (1988). El suero antiofidico polivalente producido en Costa Rica. Estabilidad y capacidad neutralizante. Revista Costarricense de Ciencias Médicas, 9(2): 155-169.

Habib, A. G. (octubre, 2013). Public health aspects of snakebite care in West Africa: perspectives from Nigeria. Journal of Venomous Animals and Toxins including Tropical Diseases, 19.

Jociles, M. I. (2003). El análisis de discurso: de cómo utilizar desde la antropología social la propuesta de Jesús Ibáñez. Ateneo de Antropología, 11(42), 1-25.

Jociles, M. I. (1994). Etnografía de un caso de "alta tecnología": sobre Aramis. Revista Política y Sociedad, 14(15): 77-97.

Rojas, G., Jiménez, J.M. y Gutiérrez, J.M. (1994). Caprylic acid fractionation of hyperimmune horse plasma: description of a simple procedure for antivenom production. Toxicon 32, 351-363.

\section{TEXTOS ELECTRÓNICOS}

Gutiérrez, J. M.; Segura, M. y Aymerich, R. (s.f). Historia del Instituto Clodomiro Picado. Recuperado de: http://cimm. ucr.ac.cr/aruiz/libros/Ciencia\%20y\%20 Tecnologia/HistoriadelaCiencialaTecnologiaylasTecnicasenCostaRica/JoseMariaGutierrez.html

Jociles, M. I. (1996). On actor-network theory. A few clarifications plus more than a few complications. Soziale Welt, 47: 369-381. Recuperado de: http://www.f.waseda.jp/ sidoli/Latour_ANT_Clarifications.pdf

Fecha de ingreso: 11/07/2016 Fecha de aprobación: 16/08/2016 
